\title{
Incremental Healthcare Costs and Outpatient Antifungal Treatment of Patients with Aspergillosis in the United States
}

\section{Emily Durden ${ }^{1 *}$, Donna McMorrow ${ }^{1}$, Paul Juneau ${ }^{1}$, Robert Fowler ${ }^{1}$, Paresh Chaudhari ${ }^{2}$, David Horn ${ }^{2}$}

${ }^{1}$ Truven Health Analytics, Austin TX, USA

${ }^{2}$ Astellas Scientific and Medical Affairs, Inc., Northbrook, IL, USA

Corresponding author: emily.durden@truvenhealth.com

\begin{abstract}
Objectives: To evaluate the total and outpatient economic burden of aspergillosis, and to describe the outpatient antifungal treatment of aspergillosis within a large, commercially-insured population in the United States.

Methods: Adults with at least one medical claim with an aspergillosis diagnosis (International Classification of Disease 9th Revision Clinical Modification [ICD-9-CM] code 117.3 or 484.6) between 07/01/04-03/01/11 were identified from the MarketScan Research Databases. Patients had $\geq 6$ months of pre-index and $\geq 1$ month of post-index continuous health plan and pharmacy benefit enrollment and no pre-index diagnosis of aspergillosis. Aspergillosis cases were propensity score-matched to a sample of controls without aspergillosis. Outpatient antifungal therapy and total and outpatient healthcare resource utilization were evaluated in the post-index period. General linear models were used to estimate costs, which were adjusted by the length of follow-up. Incremental costs were calculated between cohorts and a bootstrap procedure was used to produce corresponding variation and 95\% confidence interval estimates.
\end{abstract}

Results: Aspergillosis cases ( $\mathrm{N}=5499$; mean age: 57.8 years; $48.6 \%$ female; $64.2 \%$ with cancer) were matched to 5499 controls (mean age: 58.3 years; $48.4 \%$ female; $60.6 \%$ with cancer). Two-thirds of the aspergillosis cases had no outpatient prescription for an antifungal within 30 days of index; for those with outpatient antifungal therapy, voriconazole was the most commonly prescribed agent (60.9\%). Average adjusted total and outpatient expenditures were greater for aspergillosis patients during follow-up than those of the matched controls (\$26680 and \$9248 greater, respectively).

Conclusions: The economic burden of aspergillosis is substantial. Patients with aspergillosis utilize significantly more healthcare resources and thus incur greater healthcare costs than do similar patients without aspergillosis.

Keywords: aspergillosis, economic, burden, costs, treatment 


\section{BACKGROUND}

Aspergillosis refers to a number of diseases, including allergic bronchopulmonary aspergillosis, pulmonary aspergilloma and invasive aspergillosis, caused by infection by the commonly-encountered fungus Aspergillus. ${ }^{1}$ Though harmless to most, the fungus is responsible for considerable amounts of morbidity and mortality among immunocompromised populations, including those with chronic lung conditions, such as pneumonia and cystic fibrosis, cancer and human immunodeficiency virus (HIV), as well as those who have undergone bone marrow or solid-organ transplants. ${ }^{2}$ Case fatality is estimated to be as high as 60 to $100 \%$ in immunocompromised populations. ${ }^{2-4}$

Currently, voriconazole and amphotericin B deoxycholate (D-AMB) are the only therapies licensed in the United States for primary treatment of invasive aspergillosis. ${ }^{5}$ Voriconazole is a triazole antifungal medication administered via oral tablets or injection. Due to the superior clinical efficacy ${ }^{6}$ and safety advantages associated with its use as an initial treatment, voriconazole has emerged in recent years as the standard of care in the primary treatment of invasive aspergillosis. Other compounds (lipid formulations of AMB, itraconazole, caspofungin) are indicated as salvage therapies or for prophylaxis (posaconazole) in some transplant patients and in some cancer patients at high risk for invasive aspergillosis.,

Data show that hospitalizations for aspergillosis have increased over the last 30 years and that patients hospitalized with aspergillosis have longer average lengths of stay and higher rates of mortality than patients hospitalized with other illnesses. ${ }^{7}$ The hospital costs of patients with an aspergillosis infection vary widely depending on the severity of infection, especially in the context of comorbidities. Patients with a secondary diagnosis of aspergillosis in hospital records, presumably indicative of an immunocompromised patient, have been noted to have greater mortality, longer inpatient length of stay, longer duration of antifungal therapy and higher total hospital costs than those with a principal diagnosis of aspergillosis. ${ }^{5}$ Hospital charges for treatment of aspergillosis reported in the literature range from a median of approximately $\$ 40000$ for HIV patients ${ }^{5}$ to $\$ 413000$ for individuals with a bone marrow transplant. ${ }^{8}$ The proportion of the total hospital costs attributable to intravenous antifungal drugs is estimated to be $7.2 \% .{ }^{9}$

While aspergillosis is most often treated in the hospital setting, the availability of effective oral antifungals such as oral voriconazole may be shifting some care to the outpatient setting, whether as initial care or as continuation of therapy initiated in the hospital setting. As such, it is important to understand treatment received and costs incurred outside of the hospital. To date, most available data on the treatment and costs of aspergillosis largely focus on the hospital setting. To address the gap in the literature, the objectives of this study are twofold: 1) to describe the outpatient antifungal treatment and healthcare utilization of patients diagnosed with aspergillosis and 2) to provide recent estimates of total and outpatient healthcare costs of patients with aspergillosis in the United States.

\section{METHODS}

\section{Study Design}

This retrospective, observational study evaluates the incremental economic burden of aspergillosis within a commerciallyinsured population in the United States. Administrative claims data were used to identify a study population of enrollees (employees and their dependents) with aspergillosis and a propensity score-matched comparison group without aspergillosis. Healthcare utilization and expenditures were evaluated over a variable-length follow-up or post-index period. The economic burden of aspergillosis was estimated as the difference in the mean annualized costs between aspergillosis cases and controls, adjusted for differences in patient characteristics and variable patient follow-up.

\section{Data Sources}

All patients who met the study eligibility requirements in the Truven Health Analytics' MarketScan ${ }^{\circledR}$ Commercial Claims and Encounters (Commercial) and Medicare Supplemental and Coordination of Benefits (Medicare) Research Databases were selected into the study. The Commercial database includes medical and pharmacy claims data from a variety of fee-for-service and managed care health plans, including exclusive provider organizations 
(EPO), preferred provider organizations (PPO), point of service plans (POS), indemnity plans, health maintenance organizations (HMO), and most recently consumer driven health plans (CDHP). The Medicare database includes data from both Medicare-covered and employer-covered payments. These databases include enrollment data from approximately 150 large employers and health plans across the United States, which provide private healthcare coverage. More than 60 million employees, dependents, and retirees are included in these databases in the last three years. Both the MarketScan Commercial and Medicare Databases provide detailed cost, use, and outcomes data for healthcare services provided in both the inpatient and outpatient settings. The inpatient and outpatient medical claims are linked to outpatient pharmacy claims and person-level enrollment data through the use of unique enrollee identifiers.

\section{Study Sample and Inclusion Criteria}

Patients included in the aspergillosis study population were adults age 18 and older with at least one inpatient admission, emergency room (ER) or outpatient visit with a diagnosis of aspergillosis (International Classification of Diseases, $9^{\text {th }}$ Revision, Clinical Modification diagnosis code 117.3 or 484.6) between July 1, 2004 and March 1, 2011. The patient's index date was the date of the first claim indicating a diagnosis of aspergillosis during the study period. All patients were required to have at least six months of continuous pre-index and at least one month of continuous post-index health plan and pharmacy benefit enrollment. Patients with a diagnosis of aspergillosis in the pre-index period were excluded. To maximize use of available data, the follow-up period was variable in length, and patients were followed until the end of their continuous enrollment or March 31, 2011, whichever occurred first.

A sample of control patients without aspergillosis who also met the inclusion criteria were matched to aspergillosis patients using propensity score matching. Potential controls were selected from a 1:1000 demographically similar sample (matched on index year, age group, sex, region, and year of follow-up) of health plan enrollees without aspergillosis during the study period. The 5573 aspergillosis cases and 4932497 potential control patients were included in the propensity score matching process (described in detail below). Following the matching process, 5499 aspergillosis cases and an equal number of controls were included in the study population.

\section{Variable Definitions and Propensity Score Matching}

Patient demographic and clinical characteristics, identified during the 6-month baseline period, included age (as a continuous measure and categorized as follows: 18-34, 35-44, 45-54, 55-64, 65-74, and 75+), sex, urbanicity, U.S. Census region (South, Northeast, North Central, West, or data unavailable/unknown), index year (2004 to 2011), and health plan type (indemnity plans, PPO or POS plan, HMO and other capitated plans, high deductible health plans, and other). Index year was assigned based on the year of the patient's first observed aspergillosis diagnosis meeting all other inclusion criteria during the study period for the aspergillosis cases; for non-aspergillosis cases, the index date was randomly assigned based on the distribution of index dates in the aspergillosis cohort. A Charlson comorbidity index $(\mathrm{CCI})^{10,11}$ score (Deyo version) was calculated for the baseline period. The CCI estimates the burden of comorbid illness from diagnoses associated with chronic diseases, with higher scores indicating a greater probability of major disability or death due to comorbid illness in the 12 months after it was evaluated. In addition, a series of flags were created to identify underlying immunocompromising conditions observed during the pre- and separately, the pre- or post-index periods as follows: cancer, bone marrow transplant, hematopoietic stem cell transplant (HSCT), human immunodeficiency virus (HIV), lung transplant, and neutropenia. Flags were also created to indicate the presence of graft-versus-host disease (GVHD), diabetes, and pulmonary diseases during the pre- or post-index periods. Pre-index utilization of medications in the following classes was also evaluated: systemic corticosteroids, immunosuppressive medications, warfarin, and broad spectrum antibiotics. Finally, total healthcare expenditures during the pre-index period were calculated.

Propensity score matching was used to ensure a similar distribution of specific confounding characteristics between aspergillosis patients and a sample of matched controls. ${ }^{12,13}$ The matching variables were: age group, gender, Census region, index year, the presence of a pre-index diagnosis of cancer, bone marrow transplant, HSCT, lung transplant, neutropenia, or HIV, total pre-index healthcare costs, and number of days of follow-up. To perform the propensity score matching, logistic regression was used to predict the probability of having aspergillosis, using the values of various matching variables. Aspergillosis and non- aspergillosis patients were matched separately within the Commercial and 
Medicare cohorts on the basis of the propensity score using a nearest neighbor metric to establish the final 1:1 match. Standardized differences (a function of mean values and standard deviations in the cases and controls) less than 10 for the majority of matching factors were considered an acceptable match. ${ }^{14}$

\section{Outcome Variables and Statistical Analyses}

Outpatient utilization during the post-index period of antifungal therapy, which may be used to treat patients with aspergillosis (amphotericin B, an echinocandin (anidulafungin, caspofungin, micafungin), itraconazole, posaconazole, and voriconazole) was evaluated, as was duration (in days) of the first observed antifungal therapy. Concomitant medication use was also evaluated, including use of systemic corticosteroids, immunosuppressive agents, broad spectrum antibiotics and chemotherapeutic agents (see Table 3).

Healthcare utilization and expenditures were evaluated for the post-index period for aspergillosis cases and matched controls. Total healthcare expenditures in the post-index period included payments for inpatient admissions, outpatient visits and services (including ER visits, outpatient office visits, laboratory and radiology claims, and other outpatient services), and outpatient prescription drugs. Utilization and expenditures within each of these categories were also evaluated separately. All dollar metrics were inflated to 2011 values using the Medical Care Component of the Consumer Price Index (CPI). Healthcare utilization and expenditures were evaluated for the variable-length follow-up and results are reported both without adjustment for length of follow-up and in terms of per patient per month (PPPM).

Medical expenditures were first transformed using a common $\left(\log _{10}\right)$ transformation. Generalized linear models (GLM) were used to adjust for any remaining imbalances from matching to estimate the effects of aspergillosis diagnosis on $\log _{10}$ transformed total and outpatient healthcare expenditures. As the length of patient follow-up in this study was variable, it was necessary to accommodate this feature of the study and reduce the potential bias that could be introduced by using a summary of costs, post index..$^{15}$

A variation on a procedure suggested by Etzioni, et al., ${ }^{16}$ was employed to appropriately adjust the costs under the condition of incomplete or varying follow-up. Briefly, the probability of remaining in the study to the beginning of a time interval was calculated with a Kaplan-Meier/Product Limit estimator and then multiplied by average costs over the interval (back-transformed to the original or dollar scale using a method suggested by Dambolena, et al. ${ }^{17}$ conditional on remaining in the study to the start of the interval. The key independent variable was an indicator for aspergillosis status. The control variables included: age, gender, insurance type, region, index year, pre-index CCI score, number of unique ICD-9-CM diagnosis codes in the pre-index period, underlying immunocompromising conditions in the pre-index period (cancer, bone marrow transplant, HSCT, lung transplant, neutropenia, HIV), total medical costs in pre-period and number of days of follow-up. Patients without aspergillosis served as the reference group; the effects of an aspergillosis diagnosis on transformed total and outpatient healthcare costs were assessed relative to controls without aspergillosis.

\section{RESULTS}

The demographic and clinical characteristics of aspergillosis cases and the propensity score-matched controls $(\mathrm{n}=5499$ for each cohort) are presented in Tables 1 and 2, respectively. As shown in the tables, the propensity score matching procedure resulted in comparable groups with only one of the matching variables yielding a standardized difference of $>10$ between the cases and controls (the standardized difference for age within the Medicare study population was 11). Post-matching, the average age of the aspergillosis study cohort was $57.8 \pm 15.3$ years, and $48.6 \%$ were female. Onethird ( $\mathrm{n}=1860)$ of the overall case study population was identified from the Medicare Database and 95\% ( $\mathrm{n}=1762)$ of these patients were aged 65 and older. Two-thirds of the aspergillosis cases had their index diagnosis in the outpatient setting. The three most common immunocompromising comorbidities observed during the pre- or post-index periods for aspergillosis cases and controls, respectively, were cancer $(64.2 \%$ and $60.6 \%)$, pulmonary diseases (63.4\% and $27.8 \%)$ and diabetes $(24.6 \%$ and $26.9 \%)$. 


\section{Outpatient Treatment}

Table 3 presents the outpatient prescription drug utilization of the study population during the evaluation period. Initial outpatient antifungal therapy observed within 30 days of the index diagnosis included: voriconazole (19.8\%), itraconazole (7.5\%), an echinocandin (i.e., caspofungin, micafungin, or anidulafungin) (2.4\%), amphotericin B $(1.5 \%)$ and posaconazole $(0.90 \%)$. Few patients were treated with more than one antifungal within 30 days of index $(0.42 \%)$, while no outpatient antifungal therapy within 30 days of the index diagnosis was observed for more than two-thirds of the study population $(67.5 \%)$. One-quarter $(25.2 \%)$ of the aspergillosis study population had at least one prescription claim for an antifungal after the 30 day period following index. The average duration of antifungal therapy during the postindex period was $51.8 \pm 80.1$ days. Initial outpatient treatment with voriconazole was significantly longer (mean $=60.6$ \pm 91.1 days; $\mathrm{p}<0.001)$ than outpatient treatment with amphotericin $\mathrm{B}(19.6 \pm 37.4)$ and the echinocandins $(4.5 \pm 12.9)$ and similar to the duration of treatment with posaconazole $(47.1 \pm 51.0)$ and itraconazole $(53.6 \pm 63.3)$ (data not shown, available from the authors upon request). Nearly three-fourths $(73.0 \%)$ of the aspergillosis study population had at least one outpatient prescription claim for a systemic corticosteroid during the post-index period, $35.3 \%$ had at least one claim for a chemotherapeutic agent, and $11.2 \%$ had at least one claim for an immunosuppressive medication.

Table 1. Characteristics of Aspergillosis Cases and Propensity Score-Matched Controls

\begin{tabular}{|c|c|c|c|}
\hline & Cases & Controls & p-values \\
\hline Number of patients & 5,499 & 5,499 & \\
\hline Age, in years (mean, SD) & $57.8( \pm 15.3)$ & $58.3( \pm 15.4)$ & 0.088 \\
\hline Female $(\%)^{\dagger}$ & $48.60 \%$ & $48.40 \%$ & 0.79 \\
\hline Geographic Region $(\%)^{\dagger}$ & & & 0.87 \\
\hline Northeast & $9.00 \%$ & $8.90 \%$ & \\
\hline North Central & $28.10 \%$ & $28.00 \%$ & \\
\hline South & $36.60 \%$ & $37.40 \%$ & \\
\hline West & $25.40 \%$ & $25.00 \%$ & \\
\hline Unknown & $0.90 \%$ & $0.70 \%$ & \\
\hline Urban Residence (\%) & $85.50 \%$ & $82.80 \%$ & 0.0002 \\
\hline Health Plan Type (\%) & & & 0.92 \\
\hline Indemnity plan & $20.50 \%$ & $20.30 \%$ & \\
\hline $\mathrm{HMO} /$ other capitated plan & $17.80 \%$ & $17.50 \%$ & \\
\hline $\mathrm{PPO} / \mathrm{POS}$ & $57.10 \%$ & $57.50 \%$ & \\
\hline High deductible plan & $2.60 \%$ & $2.80 \%$ & \\
\hline Other/unknown & $1.90 \%$ & $1.90 \%$ & \\
\hline Index Year $(\%)^{\dagger}$ & & & 0.74 \\
\hline 2004 & $8.60 \%$ & $8.40 \%$ & \\
\hline 2005 & $13.00 \%$ & $13.60 \%$ & \\
\hline 2006 & $9.80 \%$ & $9.10 \%$ & \\
\hline 2007 & $13.30 \%$ & $12.90 \%$ & \\
\hline 2008 & $17.10 \%$ & $17.20 \%$ & \\
\hline 2009 & $17.90 \%$ & $17.50 \%$ & \\
\hline 2010 & $18.00 \%$ & $18.70 \%$ & \\
\hline 2011 & $2.20 \%$ & $2.40 \%$ & \\
\hline
\end{tabular}

$\mathrm{HMO}=$ health maintenance organization; $\mathrm{PPO}=$ preferred provider organization; $\mathrm{POS}=$ point of service plan; ${ }^{\dagger}$

Variables included in the propensity score matching algorithm; all variables had standardized differences of less than 10 between the aspergillosis cases and controls with the exception of age for the Medicare population (standardized difference $=11$ ). 
Table 2. Pre-Index Clinical Characteristics of Aspergillosis Cases and Propensity Score-Matched Controls

\begin{tabular}{|c|c|c|c|}
\hline & Cases & Controls & p-values \\
\hline Pre-Index CCI score (mean, SD) & $2.0( \pm 2.3)$ & $2.0( \pm 2.6)$ & 0.93 \\
\hline Unique ICD-9-CM diagnosis codes (mean, SD) & $15.2( \pm 10.8)$ & $12.5( \pm 10.1)$ & $<0.0001$ \\
\hline \multicolumn{4}{|l|}{ Pre-index comorbidities $(\%)^{\dagger}$} \\
\hline Cancer & $39.10 \%$ & $40.30 \%$ & 0.192 \\
\hline Bone marrow transplant & $2.30 \%$ & $2.40 \%$ & 0.704 \\
\hline HSCT & $0.90 \%$ & $0.90 \%$ & 0.839 \\
\hline HIV & $0.90 \%$ & $1.00 \%$ & 0.922 \\
\hline Lung transplant & $3.00 \%$ & $2.90 \%$ & 0.734 \\
\hline Neutropenia & $13.30 \%$ & $14.90 \%$ & 0.016 \\
\hline \multicolumn{4}{|l|}{ Pre- or post-index comorbidities (\%) } \\
\hline Cancer & $64.20 \%$ & $60.60 \%$ & $<0.0001$ \\
\hline Bone marrow transplant & $5.20 \%$ & $3.10 \%$ & $<0.0001$ \\
\hline HSCT & $2.00 \%$ & $1.30 \%$ & 0.0058 \\
\hline HIV & $1.20 \%$ & $1.10 \%$ & 0.591 \\
\hline Lung transplant & $8.50 \%$ & $4.80 \%$ & $<0.0001$ \\
\hline Neutropenia & $20.40 \%$ & $17.80 \%$ & 0.0007 \\
\hline GVHD & $1.90 \%$ & $0.90 \%$ & $<0.0001$ \\
\hline Diabetes & $24.60 \%$ & $26.90 \%$ & 0.0056 \\
\hline Pulmonary diseases & $63.40 \%$ & $27.80 \%$ & $<0.0001$ \\
\hline \multicolumn{4}{|l|}{ Pre-index medication use $(\%)$} \\
\hline Systemic corticosteroids & $67.30 \%$ & $40.70 \%$ & $<0.0001$ \\
\hline Immunosuppressive medications & $9.80 \%$ & $6.00 \%$ & $<0.0001$ \\
\hline Warfarin & $6.80 \%$ & $9.70 \%$ & $<0.0001$ \\
\hline Broad spectrum antibiotics & $65.40 \%$ & $43.30 \%$ & $<0.0001$ \\
\hline Total costs in pre-index period (mean, SD) ${ }^{\dagger}$ & $\$ 67752.7( \pm \$ 132666)$ & $\$ 69376.7( \pm \$ 218034)$ & 0.637 \\
\hline
\end{tabular}

$\mathrm{SD}=$ standard deviation; $\mathrm{CCI}=$ Charlson Comorbidity Index; HSCT=hematopoietic stem cell transplantation; HIV=human immunodeficiency virus; GVHD=graft-versus-host disease; †Variables included in the propensity score matching algorithm; all variables had standardized differences of less than 10 between the aspergillosis cases and controls

\section{Healthcare Utilization and Unadjusted Healthcare Expenditures}

As shown in Table 4, a higher proportion of aspergillosis cases had an inpatient admission (49.5\% vs. 37.1\%), as well as a longer average length of stay ( $9.5 \pm 13.6$ vs. $8.0 \pm 12.8$ days), during follow-up than patients without aspergillosis. Aspergillosis cases also had a higher rate of ER visits (50.7\% vs. $42.3 \%)$ and more per PPPM outpatient office visits $(1.3 \pm 1.4$ vs. $0.9 \pm 1.1)$, laboratory tests $(4.0 \pm 11.0$ vs. $2.4 \pm 5.8)$ and outpatient prescriptions $(3.8 \pm 3.4$ vs. $3.1 \pm 2.9)$ compared to patients without aspergillosis. All comparisons were significant at $\mathrm{p}<0.001$.

As shown in Table 5, patients with aspergillosis had significantly higher PPPM expenditures in the post-index period compared to matched controls in every category of service. Mean unadjusted total PPPM healthcare expenditures were $\$ 8840 \pm \$ 21853$ for aspergillosis cases, compared to $\$ 5726 \pm \$ 26569$ for the controls, while the mean unadjusted PPPM outpatient prescription expenditures were $\$ 881 \pm \$ 1361$ for the aspergillosis cases, compared to $\$ 517 \pm \$ 1663$ for the controls. Outpatient costs (visits, services, and prescriptions) accounted for approximately $44 \%$ of the total costs of patients with aspergillosis. 
Table 3. Post-Index Outpatient Antifungal and Other Therapies of Aspergillosis Cases

\begin{tabular}{|c|c|}
\hline & $\% /$ Mean (SD) \\
\hline \multicolumn{2}{|l|}{ Antifungal treatment in first 30 days of index (\%) } \\
\hline Amphotericin B & $1.50 \%$ \\
\hline Voriconazole & $19.80 \%$ \\
\hline Itraconazole & $7.50 \%$ \\
\hline Posaconazole & $0.90 \%$ \\
\hline \multicolumn{2}{|l|}{ Echinocandins } \\
\hline Caspofungin & $1.90 \%$ \\
\hline Micafungin & $0.50 \%$ \\
\hline Anidulafungin & $0.10 \%$ \\
\hline Treated with more than 1 Antifungal at index & $0.40 \%$ \\
\hline No observed treatment at index & $67.50 \%$ \\
\hline Duration of first observed antifungal therapy (mean, SD) & $51.8(80.1)$ \\
\hline Antifungal therapy $>30$ days of index $(\%)$ & $25.20 \%$ \\
\hline Amphotericin B & $2.20 \%$ \\
\hline Voriconazole & $15.50 \%$ \\
\hline Itraconazole & $7.90 \%$ \\
\hline Posaconazole & $2.50 \%$ \\
\hline \multicolumn{2}{|l|}{ Echinocandins } \\
\hline Caspofungin & $1.90 \%$ \\
\hline Micafungin & $0.80 \%$ \\
\hline Anidulafungin & $0.10 \%$ \\
\hline \multicolumn{2}{|l|}{ Concomitant Medications, post-index period (\%) } \\
\hline Amiodarone & $2.10 \%$ \\
\hline Anti-retroviral therapy & $0.90 \%$ \\
\hline Antituberculosis medications & $4.00 \%$ \\
\hline Chemotherapeutic agents & $35.30 \%$ \\
\hline Immunosuppressive medications & $11.20 \%$ \\
\hline Phenobarbituates & $0.80 \%$ \\
\hline Statins & $27.00 \%$ \\
\hline Systemic corticosteroids & $73.00 \%$ \\
\hline Warfarin & $9.50 \%$ \\
\hline Therapeutic Drug Monitoring (\%) & $24.20 \%$ \\
\hline
\end{tabular}

$\mathrm{SD}=$ standard deviation 
Table 4. Healthcare Utilization of Aspergillosis Cases and Propensity-Score Matched Controls

\begin{tabular}{|c|c|c|c|}
\hline & Cases & Controls & p-values \\
\hline \multicolumn{4}{|l|}{ Inpatient Admissions } \\
\hline Patients with an admission (\%) & $49.50 \%$ & $37.10 \%$ & $<0.0001$ \\
\hline PPPM number of admissions (mean, SD) & $0.1( \pm 0.2)$ & $0.1( \pm 0.2)$ & $<0.0001$ \\
\hline Number of inpatient admissions (mean, SD) & $1.4( \pm 2.4)$ & $0.9( \pm 1.8)$ & $<0.0001$ \\
\hline Length of stay (mean, SD) & $9.5( \pm 13.6)$ & $8.0( \pm 12.8)$ & $<0.0001$ \\
\hline \multicolumn{4}{|l|}{ Emergency Department Visits } \\
\hline Patients with an ER visit (\%) & $50.70 \%$ & $42.30 \%$ & $<0.0001$ \\
\hline PPPM number of ER visits (mean, SD) & $0.1( \pm 0.3)$ & $0.1( \pm 0.3)$ & $<0.0001$ \\
\hline Number of ER visits (mean, SD) & $1.9( \pm 4.7)$ & $1.4( \pm 7.2)$ & $<0.0001$ \\
\hline \multicolumn{4}{|l|}{ Outpatient Physician Office Visits } \\
\hline Patients with a visit $(\%)$ & $91.60 \%$ & $90.50 \%$ & 0.0386 \\
\hline PPPM number of visits (mean, SD) & $1.3( \pm 1.4)$ & $0.9( \pm 1.1)$ & $<0.0001$ \\
\hline Number of visits (mean, SD) & $28.0( \pm 34.3)$ & $20.6( \pm 27.9)$ & $<0.0001$ \\
\hline \multicolumn{4}{|l|}{ Ophthalmology visits } \\
\hline Patients with a visit $(\%)$ & $14.40 \%$ & $11.70 \%$ & $<0.0001$ \\
\hline PPPM number of visits (mean, SD) & $0.0( \pm 0.1)$ & $0.0( \pm 0.1)$ & 0.0541 \\
\hline Number of visits (mean, SD) & $0.4( \pm 1.4)$ & $0.3( \pm 1.5)$ & 0.1271 \\
\hline \multicolumn{4}{|l|}{ Laboratory Tests } \\
\hline Patients with a laboratory test (\%) & $87.50 \%$ & $83.20 \%$ & $<0.0001$ \\
\hline PPPM number of laboratory tests (mean, SD) & $4.0( \pm 11.0)$ & $2.4( \pm 5.8)$ & $<0.0001$ \\
\hline Number of laboratory tests (mean, SD) & $60.1( \pm 137.6)$ & $38.0( \pm 78.3)$ & $<0.0001$ \\
\hline \multicolumn{4}{|l|}{ Outpatient Prescriptions } \\
\hline Patients with an outpatient prescription (\%) & $91.80 \%$ & $93.00 \%$ & 0.0130 \\
\hline PPPM number of prescriptions (mean, SD) & $3.8( \pm 3.4)$ & $3.1( \pm 2.9)$ & $<0.0001$ \\
\hline Number of prescriptions (mean, SD) & $88.2( \pm 116.3)$ & $72.8( \pm 102.5)$ & $<0.0001$ \\
\hline
\end{tabular}

PPPM=Per Patient Per Month; $\mathrm{SD}=$ standard deviation 
Table 5. Unadjusted Healthcare Expenditures of Aspergillosis Cases and Matched Controls

\begin{tabular}{|c|c|c|c|c|c|}
\hline & \multicolumn{2}{|c|}{ Cases } & \multicolumn{2}{|c|}{ Controls } & \multirow[b]{2}{*}{ p-value } \\
\hline & Mean & SD & Mean & SD & \\
\hline Inpatient admissions, total & $\$ 46034$ & $\$ 126855$ & $\$ 24273$ & $\$ 81887$ & $<0.0001$ \\
\hline PPPM & $\$ 4664$ & $\$ 17583$ & $\$ 2292$ & $\$ 10676$ & $<0.0001$ \\
\hline Emergency department & $\$ 1058$ & $\$ 5989$ & $\$ 759$ & $\$ 3235$ & 0.0010 \\
\hline PPPM & $\$ 65$ & $\$ 331$ & $\$ 46$ & $\$ 181$ & $<0.0001$ \\
\hline Outpatient physician office visits & $\$ 2891$ & $\$ 3992$ & $\$ 2076$ & $\$ 3375$ & $<0.0001$ \\
\hline PPPM & $\$ 143$ & $\$ 212$ & $\$ 98$ & $\$ 151$ & $<0.0001$ \\
\hline Ophthalmology visits & $\$ 38$ & $\$ 138$ & $\$ 32$ & $\$ 144$ & 0.0270 \\
\hline PPPM & $\$ 2$ & $\$ 8$ & $\$ 1$ & $\$ 6$ & 0.0070 \\
\hline Laboratory tests & $\$ 4444$ & $\$ 14978$ & $\$ 2140$ & $\$ 5766$ & $<0.0001$ \\
\hline PPPM & $\$ 345$ & $\$ 1,717$ & $\$ 150$ & $\$ 505$ & $<0.0001$ \\
\hline Other outpatient services & $\$ 33826$ & $\$ 81888$ & $\$ 31818$ & $\$ 129366$ & 0.3310 \\
\hline PPPM & $\$ 2494$ & $\$ 7471$ & $\$ 2637$ & $\$ 23528$ & 0.7000 \\
\hline Outpatient Prescriptions & $\$ 18490$ & $\$ 34486$ & $\$ 11152$ & $\$ 29083$ & $<0.0001$ \\
\hline PPPM & $\$ 881$ & $\$ 1361$ & $\$ 517$ & $\$ 1663$ & $<0.0001$ \\
\hline Antifungals & $\$ 5943$ & $\$ 20740$ & $\$ 263$ & $\$ 3256$ & $<0.0001$ \\
\hline PPPM & $\$ 451$ & $\$ 1712$ & $\$ 18$ & $\$ 210$ & $<0.0001$ \\
\hline Total medical expenditures & $\$ 110686$ & $\$ 196912$ & $\$ 75961$ & $\$ 178360$ & $<0.0001$ \\
\hline PPPM & $\$ 8840$ & $\$ 21853$ & $\$ 5726$ & $\$ 26569$ & $<0.0001$ \\
\hline
\end{tabular}

Notes: Expenditures were accumulated in the variable-length follow-up period of at least 1 month. With the exception of the costs of other outpatient services and ophthalmology visits, all point estimates for aspergillosis cases were significantly higher than the controls at $\mathrm{p}<0.001 ; \mathrm{SD}=$ standard deviation; $\mathrm{PPPM}=$ per patient per month

\section{Adjusted Healthcare Expenditures}

Multivariate-adjusted mean and incremental total and outpatient healthcare expenditures are shown in Table 6. After adjusting for any remaining imbalances following propensity score matching, GLM models showed total adjusted annualized healthcare expenditures were $\$ 72712.85$ among aspergillosis cases compared to $\$ 46032.63$ for the controls (incremental costs: \$26680.21). Total adjusted annualized outpatient expenditures were $\$ 31423.69$ among aspergillosis cases compared to $\$ 22175.81$ for the controls (incremental costs: $\$ 9247.88$ ).

Table 6. Multivariate-adjusted ${ }^{*}$ Total and Outpatient Costs of Patients with Aspergillosis

\begin{tabular}{|c|c|c|c|c|}
\hline & Mean & SE & 95\% Lower Confidence Limit & 95\% Upper Confidence Limit \\
\hline \multicolumn{5}{|l|}{ Total Costs } \\
\hline Aspergillosis cases & $\$ 72712.85$ & $\$ 1282.99$ & $\$ 70380.19$ & $\$ 75362.39$ \\
\hline Matched controls & $\$ 46032.63$ & $\$ 820.45$ & $\$ 44412.43$ & $\$ 47683.69$ \\
\hline Incremental costs & $\$ 26680.21$ & $\$ 1417.33$ & $\$ 23954.10$ & $\$ 29616.82$ \\
\hline \multicolumn{5}{|l|}{ Total Outpatient Costs } \\
\hline Aspergillosis cases & $\$ 31423.69$ & $\$ 657.44$ & $\$ 23169.61$ & $\$ 32810.05$ \\
\hline Matched controls & $\$ 22175.81$ & $\$ 515.45$ & $\$ 21177.76$ & $\$ 23169.61$ \\
\hline Incremental costs & $\$ 9247.88$ & $\$ 766.25$ & $\$ 7829.48$ & $\$ 10878.76$ \\
\hline
\end{tabular}

*Models adjusted for age group, gender, health plan type, region, index year, pre-index CCI score, number of unique three-digit ICD-9-CM codes, total medical costs in pre-index period, number of days of follow-up, and a series of comorbidities: cancer, bone marrow transplant, hematopoietic stem cell transplant, lung transplant, graft-versus-host disease, neutropenia, HIV, diabetes and pulmonary diseases; $\mathrm{SE}=$ standard error 
The estimated median incremental total costs of patients with aspergillosis, adjusted for the probability of remaining in the study for a given duration of follow-up (via the Kaplan Meier/Product Limit estimator), were \$22 732.99, \$19620.51 and $\$ 17870.09$ for durations of 90, 180, and 365 days of follow-up, respectively (see Figure 1). The estimated median incremental total outpatient costs of patients with aspergillosis, which were adjusted for the probability of remaining in the study for a given duration of follow-up time (via the Kaplan Meier/Product Limit estimator), were $\$ 7686.37$, $\$ 6631.56$ and $\$ 6120.40$ for durations of 90,180 , and 365 days, respectively. Upon first consideration, the estimates of costs, adjusted for varying patient follow-up time via the method suggested by Etzioni, et al., may seem counterintuitive insofar as the costs appear to decrease over time $(\$ 22732.99, \$ 19620.51$ and $\$ 17870.09$ for durations of 90,180 , and 365 days, respectively). These adjusted costs are estimated by the product of two factors. The first is the probability of remaining in the study to the next time interval. This quantity, by definition, will decline over time with loss to follow-up. The second is the costs averaged over a particular interval.

With aspergillosis, the largest changes in a patient's costs occur early (post-index), and then increase more slowly as a person will either succumb to consequences of the infection or are successfully treated. Thus, the adjusted costs, as a product of these two factors, represents a conflict between two quantities moving in opposing directions as time moves forward. If the rate at which patients exit the study grows more quickly than the rate of change in the costs as time moves forward, it becomes clear that the product, the adjusted costs, will decline (i.e., the probability, a number less than one, continues to decrease faster towards zero than the costs can accumulate away from their initial high value).

Figure 1. Total Healthcare Costs Adjusted for Covariates and Length of Follow-up Probability

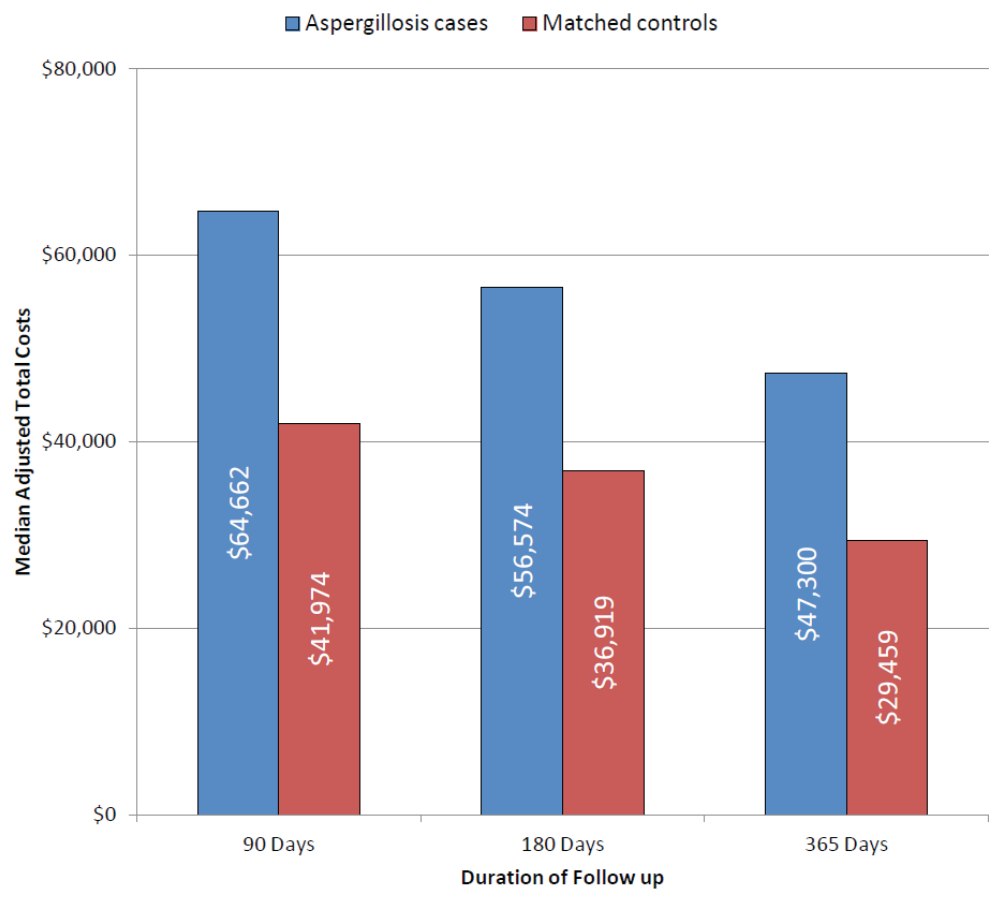

\section{DISCUSSION}

This retrospective database analysis evaluated the total direct cost burden (i.e., inpatient and outpatient) and the outpatient antifungal treatment of patients with aspergillosis within a commercially-insured population in the United States. Healthcare expenditure, both total and outpatient expenditure, were substantially higher for patients with aspergillosis compared to those without. Using propensity score matched cohorts, GLM models showed that adjusted annualized incremental total and outpatient costs of patients with aspergillosis were $\$ 26680.21$ and $\$ 9247.88$, respectively. As for outpatient antifungal therapy, we observed no outpatient prescription claim for an antifungal therapy within 30 days of the index diagnosis for two-thirds of the aspergillosis cases in our study; for those who did receive outpatient prescription therapy, voriconazole was the most commonly prescribed antifungal agent $(60.9 \%$ of those treated). 
This study provides recent estimates of the total cost burden of aspergillosis in the United States. Further, to our knowledge, it is the first to evaluate the total per patient outpatient burden, including physician office visits and laboratory and radiology services for patients with aspergillosis. Estimates of the outpatient costs of aspergillosis are important as treatment of the illness spreads to the outpatient setting with the availability of convenient, safe, and effective oral antifungal agents.

\section{Limitations}

Certain limitations inherent to retrospective analyses using administrative claims data must be considered when interpreting these results. First, potential miscoding of medical claims and missing data are possible. Administrative data are collected for financial and administrative rather than research purposes, which can lead to suboptimal sensitivity and specificity when measuring conditions. Claims data arise from a person's use of a healthcare system and therefore depend on reimbursement coding practices from physician office visits, prescriptions filled and hospital stays. The second limitation is with respect to representativeness - while the MarketScan Research Databases represent a wide variety of nationally-representative employed patients and their dependents, it is not a random sample. The study population includes patients with Commercial and Medicare supplemental insurance, thus the results might not be generalizable to people with other insurance or with no insurance. An additional caveat to consider is that because our study design required at least 1 month of follow-up, the costs of patients who died within 1 month following diagnosis were not captured. As healthcare costs preceding death tend to be quite substantial, excluding such patients may have resulted in conservative incremental cost estimates in the present study. Finally, although we controlled for overt bias by propensity score matching aspergillosis cases and controls on a number of potentially key demographic and clinical characteristics, the two groups may differ on some unmeasured characteristics unrepresented by claims data.

\section{CONCLUSION}

The total economic burden of aspergillosis continues to be substantial. Patients with aspergillosis utilize significantly more inpatient and outpatient healthcare resources and thus incur greater healthcare expenditures than do similar patients without aspergillosis. Efforts to improve prevention, diagnosis, and treatment of aspergillosis may help to reduce the cost of care and the overall patient burden associated with this illness.

\section{CONFLICT OF INTEREST DECLARATION}

This research was sponsored by Astellas USA and was conducted by Truven Health Analytics. Publication of study results was not contingent on the sponsor's approval. Dr. Durden, Mr. Juneau, Ms. McMorrow and Mr. Fowler are employees of Truven Health Analytics; Dr. Chaudhari is an employee and stockholder of Astellas USA. Dr. Horn has received research funding from Astellas USA and has served as a consultant to and on advisory boards for Astellas USA.

\section{REFERENCES}

${ }^{1}$ Centers for Disease Control and Prevention: Aspergillosis. http://www.cdc.gov/fungal/aspergillosis/. Accessed June 2013.

${ }^{2}$ Lin S, Schranz J, Teutsch: Aspergillosis case-fatality rate: Systematic review of the literature. Clin Infect Dis 2003;32(3):35866.

${ }^{3}$ Denning DW: Invasive aspergillosis. Clin Infect Dis 1998;26(4):781-803.

${ }^{4}$ Patterson TF, Kirkpatrick WR, White M, et al: Invasive aspergillosis: Disease spectrum, treatment practices, and outcomes. I3 aspergillus study group. Medicaine (Baltimore) 2000;79(4):250-60.

${ }^{5}$ Walsh TJ, Anaissie EJ, Denning DW, et al: Treatment of aspergillosis: Clinical practice guidelines of the infectious diseases society of America. Clin Infect Dis 2008;46(3):327-60.

${ }^{6}$ Herbrecht R, Denning DW, Patterson TF, et al: Voriconazole versus amphotericin B for primary therapy of invasive aspergillosis. N Engl J Med 2002;347(6):408-15. 


\section{JHEOR}

${ }^{7}$ Dasbach EJ, Davies, GM, Teutsch SM: Burden of aspergillosis in the United States. Clin Infect Dis 2000;31(6):1524-8.

${ }^{8}$ Tong KB, Lau CJ, Murtagh K, Layton AJ, Seifeldin R: The economic impact of Aspergillosis: Analysis of hospital expenditures across patient subgroups. Int J Infect Dis 2009;13(1):24-36.

${ }^{9}$ Kim A, Nicolau DP, Kuti JL: Hospital costs and outcomes among intravenous antifungal therapies for patients with invasive aspergillosis in the United States. Mycoses 2011;54(5):e301-12.

${ }^{10}$ Charlson ME, Pompei P, Ales KL, MacKenzie CR: A new method of classifying prognostic comorbidity in longitudinal studies: development and validation. J Chronic Dis 1987;40(5):373-83.

${ }^{11}$ Romano PS, Roos LL, Jollis JG: Adapting a clinical comorbidity index for use with ICD-9-CM administrative data: differing perspectives. J Clin Epidemiol 1993;46(10):1075-9.

${ }^{12}$ Heckman JJ, Ichimura H, Todd P: Matching as an econometric evaluation estimator: Evidence from evaluating a job training programme. Rev Econ Studies 1997;64:605-54.

${ }^{13}$ Austin PC, Grootendorst P, Anderson MG: A comparison of the ability of different propensity score models to balance measured variables between treated and untreated subjects: A Monte Carlo study. Stat Med 2007;26:734-53.

${ }^{14}$ Ho DE, Imai K, King G, Stuart EA: Matching as nonparametric preprocessing for reducing model dependence in parametric causal inference. Political Analysis 2007;15:199-236.

${ }^{15}$ Lin DY, Feuer EJ, Etzioni R, Wax Y: Estimating medical costs from incomplete follow-up data. Biometrics 1997;53:41934.

${ }^{16}$ Etzioni RD, Feuer EJ, Sullivan SD, Lin D, Hu C, Ramsey SD: On the use of survival analysis techniques to estimate medical care costs. J Health Econ 1999;18(3):365-80.

${ }^{17}$ Dambolena, IG, Eriksen, SE, Kopsco, DP: Logarithmic transformations in regression: Do you transform back correctly? PRIMUS: Problems, Resources, and Issues in Mathematics Undergraduate Studies, 2009;19(3):280-95. 